\title{
A model for active and healthy ageing with a rare genetic disease: cystic fibrosis
}

\author{
Raphael Chiron ${ }^{1,2,13}$, Davide Caimmi ${ }^{1,2,3,13}$, Arunas Valiulis ${ }^{4}$, Isabelle Durieu $^{5}$, \\ Patrick Tejedor ${ }^{6}$, Yam Le Cam ${ }^{7}$, Mathieu Boudes ${ }^{7}$, Oliver Jonquet ${ }^{1}$, \\ Timo Strandberg ${ }^{8}$, Jean-Pierre Michel $^{9}$, Esteban de Manuel Keenoy ${ }^{9}$, \\ Pascal Demoly $2,3,10$, Jacques Mercier ${ }^{1,2}$, Radolphe Bourret ${ }^{2,11,12}$ and \\ Jean Bousquet ${ }^{2,12}$ on behalf of MACVIA-LR European Innovation Partnership on \\ Active and Healthy Ageing Reference Site
}

Affiliations: ${ }^{1}$ Cystic Fibrosis Center, University Hospital of Montpellier, Montpellier, France. ${ }^{2}$ MACVIA-LR, European Innovation Partnership on Active and Healthy Ageing Reference Site, University Hospital of Montpellier, Montpellier, France. ${ }^{3}$ Sorbonne Universités, UPMC Paris 06, UMR-S 1136, IPLESP, Equipe EPAR, Paris, France. ${ }^{4}$ Vilnius University Clinic of Children's Diseases, Vilnius, Lithuania. ${ }^{5}$ Adult Cystic Fibrosis Center, Groupe Hospitalier Sud, Hospices Civils de Lyon, Université de Lyon, Pierre Benite Cedex, France. ${ }^{6}$ Vaincre la mucoviscidose, Paris, France. ${ }^{7}$ EURORDIS - Rare Diseases Europe, Plateforme Maladies Rares, Paris, France. ${ }^{8}$ Geriatric Dept, University of Helsinki, Helsinki, Finland. ${ }^{9}$ University of Geneva, European Union Geriatric Medicine Society (EUGMS), Geneva, Switzerland. ${ }^{10}$ Allergy Unit, Département de Pneumologie et Addictologie, Hôpital Arnaud Villeneuve, University Hospital of Montpellier, Montpellier, France. ${ }^{11}$ INSERM, VIMA: Ageing and chronic diseases. Epidemiological and public health approaches, Paris, France. ${ }^{12}$ UVSQ, UMR-S 1168, Université Versailles St-Quentin-en-Yvelines, Paris, France. ${ }^{13}$ Both authors contributed equally to the paper.

Correspondence: Jean Bousquet, CHRU Arnaud de Villeneuve, Département de Pneumologie, 371 Avenue du Doyen Gaston Giraud, 34295 Montpellier Cedex 5, France. E-mail: jean.bousquetdorange.fr

@ERSpublications

Cystic fibrosis management today: an example of active and healthy ageing in patients suffering from a rare genetic disease http://ow.ly/V3JXt

Cystic fibrosis (CF), the most common life-limiting genetic condition in Caucasians, affects many organ systems. Specialised medical care, aggressive treatments [1] and proper nutrition both lengthen and improve quality of life for CF patients. Advances achieved over the last four decades have transformed CF from a fatal childhood disease to a long-term chronic condition. CF patients can now reach adulthood [2-5], although most of them still have a reduced life expectancy, and the adult population has passed from $44.55 \%$ in 2005 to $49.3 \%$ in 2010 (figure 1), the oldest patient being aged $>80$ years, from the 2010 European CF registry [6]. In the near future, even though CF paediatric care will remain crucial to assure the best growth for patients and transition to adulthood, CF centres may largely be considered as adult oriented [7], and we should, therefore, aim for the new concept of active and healthy ageing (AHA) in CF patients. Important controversies exist concerning the early- and long-term management of CF, which should be carefully considered when proposing a strategy for ageing well with CF [8].

Health is a multidimensional concept, capturing how people feel and function. It includes at least three dynamic components: first, survival to old age; second, delay in chronic diseases onset and disability; and third, optimal functioning for the maximal period of time. AHA includes functioning, activities and participation, diseases, and resilience (the ability to adapt). Although most AHA programmes attempt to improve old age by targeting adults, it is important to initiate AHA health promotion very early in life [9]. The European Innovation Partnership (EIP) on AHA aims to enable European Union (EU) citizens to lead healthy, active and independent lives while ageing. The Reference Site of the Languedoc Roussillon

Received: July 282015 | Accepted after revision: Oct 232015

Conflict of interest: Disclosures can be found alongside the online version of this article at erj.ersjournals.com

Copyright OERS 2016 


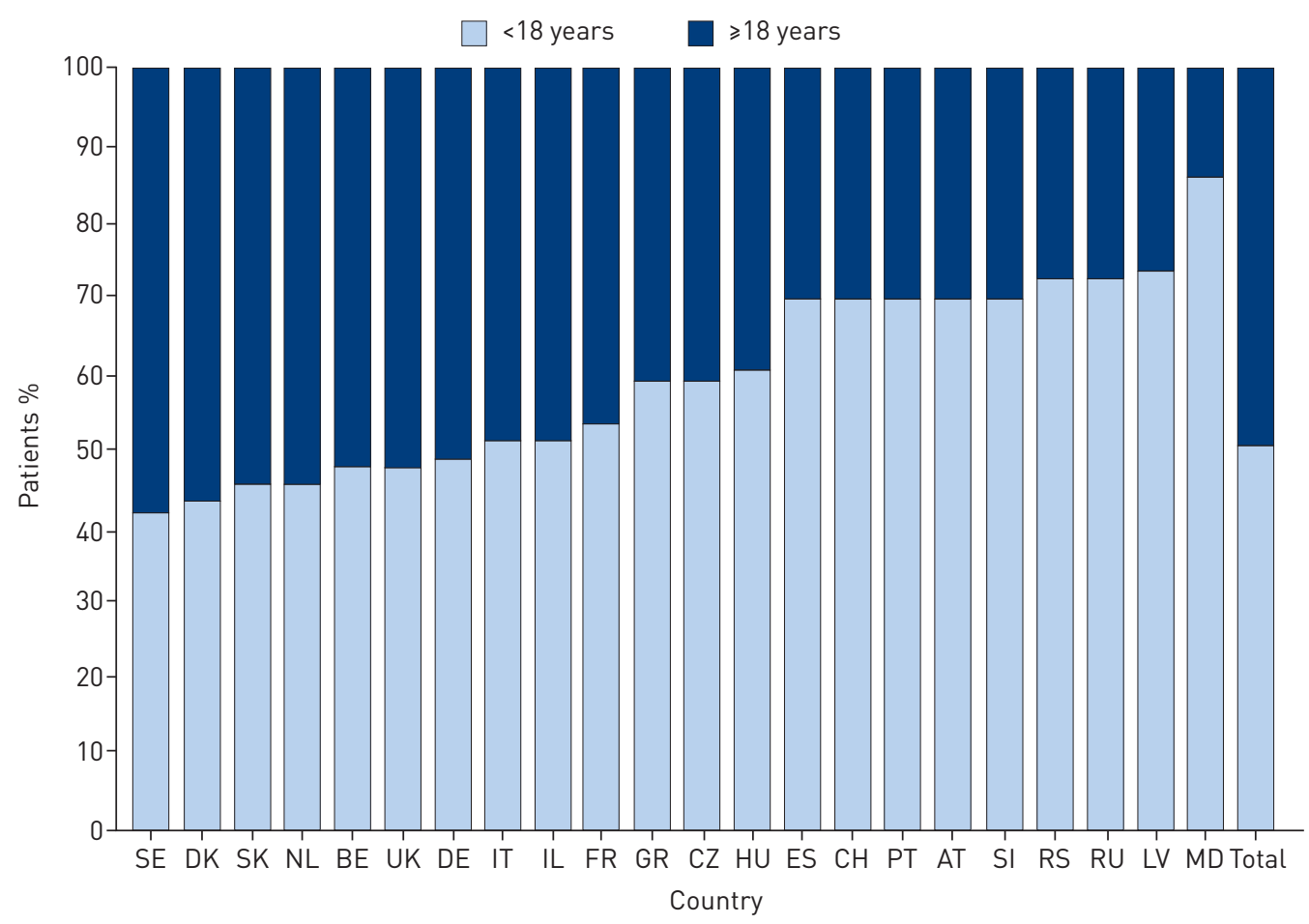

FIGURE 1 Proportion of adults aged $\geqslant 18$ years and children aged $<18$ years with cystic fibrosis who were alive on December 31, 2010. SE: Sweden; DK: Denmark; SK: Slovak Republic; NL: the Netherlands; BE: Belgium; UK: United Kingdom; DE: Germany; IT: Italy; IL: Israel; FR: France; GR: Greece; CZ: Czech Republic; HU: Hungary; ES: Spain; CH: Switzerland; PT: Portugal; AT: Austria; SI: Slovenia; RS: Serbia; RU: Russian Federation; LV: Latvia; MD: Republic of Moldova. Reproduced with permission from [6].

(MACVIA-LR) has a focus on the prevention of AHA in health and disease [10-12] with a life course perspective $[13,14]$.

Whereas in subjects without disease AHA aims to improve healthy life years, in patients with a severe disease it also focuses on increased life expectancy (figure 2). Embedding AHA in CF implies that life expectancy is not the only parameter to be considered but that every effort should be made to enhance the quality of survival.

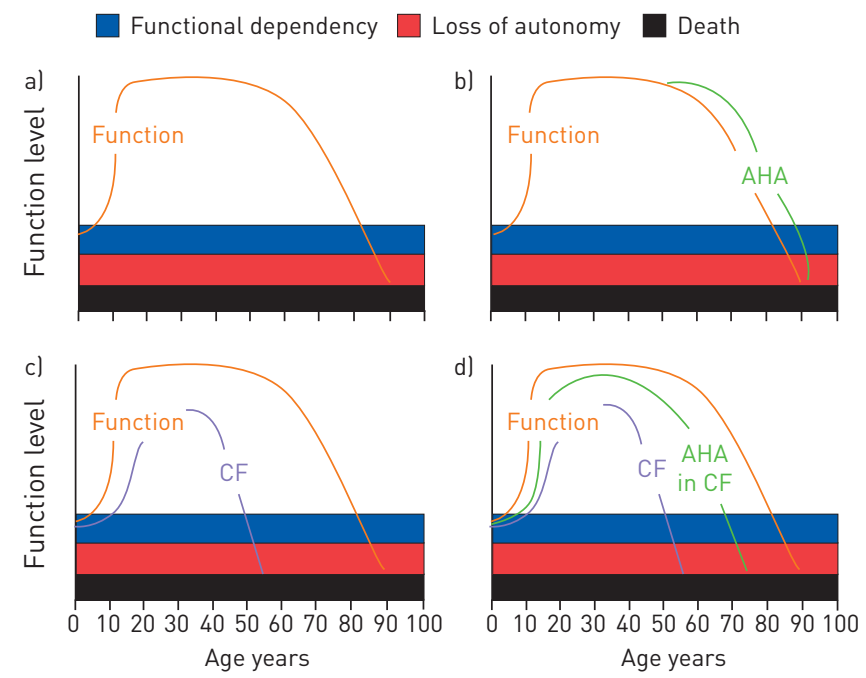

FIGURE 2 Concept of active and healthy ageing in cystic fibrosis (CF) patients. a) Functional abilities increase in childhood, reach a plateau in adulthood and decline leading to functional dependency, loss of autonomy and ultimately death. b) Active and healthy ageing (AHA) attempts to increase functional dependency and loss of autonomy by 2 years without changing life span. C) In CF patients, functional ability is not reached and patients have a shorter life expectancy. d) The concept of AHA is 1) to increase the functional ability, 2) to improve life expectancy, and 3) to delay functional dependency and loss of autonomy. 
The concept of ageing well with CF is associating the management of CF in particular and of chronic diseases in general. It includes early diagnosis, therapeutic innovation, optimal management and social care (figure 2). It is part of AIRWAYS-ICPs (Integrated care pathways for airway diseases), Action Group B3 of the EIP on AHA [15].

\section{Action plan}

\section{Screening and management}

Early detection by newborn-screening early management [16] aims to provide the potential for better pulmonary outcomes and psychosocial adaptation and has become common throughout Europe (figure 3) [6]. Screening seems less expensive than traditional diagnosis [17], but there are limitations to this approach. It is important to develop biomarkers to evaluate disease severity and prognosis. Incidental findings may occur in those with a CF gene without a CF phenotype or mild CF (e.g. male infertility). Over 2000 alterations are described in the CFTR gene (www.cftr2.org), but fewer than 150 are disease causing [18], and there are divergent opinions about how these uncertainties should be managed. A recent guideline highlights the paucity of evidence on which clinicians can base the management of screened infants in the first year of life. Finally, iatrogenic complications are common (e.g. nephrotoxic antibiotics in infancy, renal failure in adulthood, selection of resistant bacteria and antibiotic allergy) [19] and over medicalisation may present a risk of cross-infection during visits to the CF centre. One common long-term complication is loss of hearing, due to frequent aminoglycosides prescriptions and to azithromycin prescribed as a maintenance anti-inflammatory therapy. Moreover, clinicians also deal with CF patients who have been transplanted several years before and who present non-respiratory complications, since the disease shows a multisystem involvement.

\section{Innovative treatments have recently been proposed}

The abnormal CF transmembrane conductance regulator (CFTR) protein has become a therapeutic target [20] and, in the future, gene therapy may become available and help improve patients' management. Ivacaftor, a CFTR potentiator that enhances the chloride transport of function CFTR on the cell surface [21], is very effective in patients with a G551D or other gating (class III) mutation populations. However, its high cost may be an obstacle in the uptake of this treatment. The main priority for further research is the long-term effectiveness of ivacaftor [22]. Unfortunately, only few CF patients have the mutation and other targets should be developed. Lumacaftor, an investigational CFTR corrector that increases trafficking of

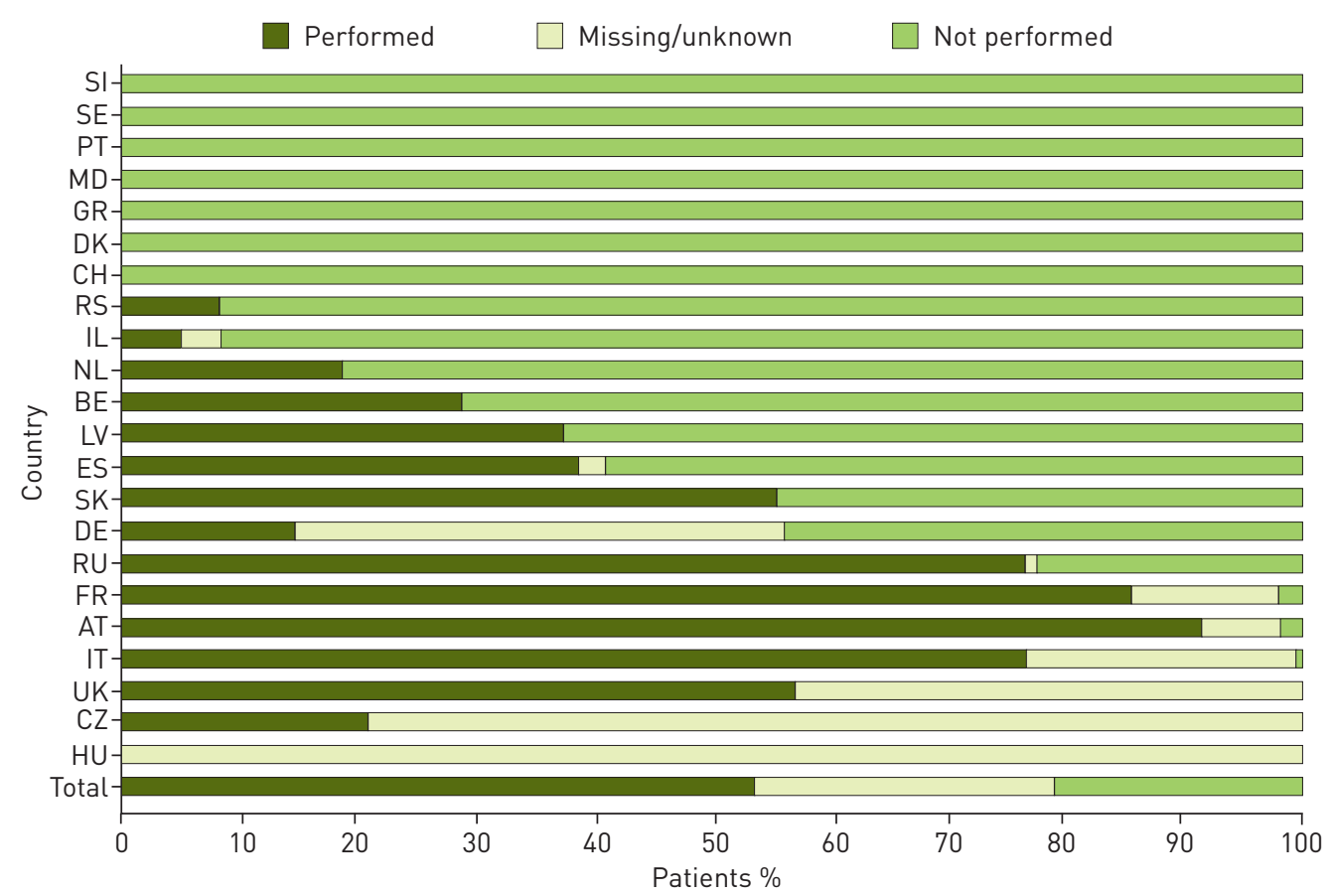

FIGURE 3 Proportion of patients who underwent neonatal screening, by country and overall. Patients aged $\leqslant 5$ years as seen in 2010. SI: Slovenia; SE: Sweden; PT: Portugal; MD: Republic of Moldova; GR: Greece; DK: Denmark; CH: Switzerland; RS: Serbia; IL: Israel; NL: the Netherlands; BE: Belgium; LV: Latvia; ES: Spain; SK: Slovak Republic; DE: Germany; RU: Russian Federation; FR: France; AT: Austria; IT: Italy; UK: United Kingdom; CZ: Czech Republic; HU: Hungary. Reproduced with permission from [6]. 
phe508del CFTR to the cell surface, when combined with ivacaftor was found to be marginally effective in homozygote phe508del [23]. However, these new therapies may have some complications in the long term (e.g. ocular abnormalities with ivacaftor), and these will then change the disease profile, as we have already seen with other innovative therapies applied to different chronic diseases.

Innovative modes for the administration of antibiotics have already improved the quality of life for $\mathrm{CF}$ patients [24] and seem to be associated with a better compliance to the prescribed treatment $[25,26]$.

\section{A holistic approach for the management of chronic diseases is needed}

$\mathrm{CF}$ is traditionally thought to affect the lungs and pancreas, but many organ systems are affected. CF comorbidities include diabetes mellitus, renal problems, cardiovascular diseases, reproductive system abnormalities and osteopenia. They increase with age. Diabetes mellitus has emerged as a common CF comorbidity and is considered a clinical entity, i.e. CF-related diabetes (CFRD), distinct from that of type I and type II diabetes [27]. With an increasing survival of patients, the impact of chronic inflammation and nutritional compromise on organ systems is increasing. For example, optimising bone health has to start in childhood (weight-bearing exercise, optimal vitamin $\mathrm{D}$ and $\mathrm{K}$ status, ingestion of dairy products, minimal steroid use, aggressive treatment of infection and inflammation) to avoid later osteopenia.

Physicians treating CF should propose a multidisciplinary approach to optimally manage CF patients. On the other hand, specialists should consider CF as the entire disease and not as separate entities to propose a holistic management.

\section{Transition from childhood to adulthood and to geriatrics}

CF patients are now living long enough to require transition from paediatric CF centres to adult $\mathrm{CF}$ centres. The goal of transition is for the young adult to be engaged in the adult health care system in ways that optimise health, maximise potential, and increase quality of life [6]. Currently, there is an information gap in the literature for psychological models that can help guide informed transition processes. There should be inter-related constructs of patients, parents and providers with emphasis on variables amenable to intervention [28]. The new challenge is, however, the transition from adult care to geriatrics. This will be done using EU-funded programmes on care pathways on the transition from adults to the elderly in order to prevent frailty such as CAREWELL (www.carewell-project.eu/home/) and ASSEHS (the Activation of Stratification Strategies and Results of the interventions on frail patients of Healthcare Services) [29].

\section{CF reference centres}

CF reference centres exist in most European countries. They are of great importance for the diagnosis and management of CF, they have a global vision of the disease and its comorbidities or complications, and they include well-established cohorts to test new strategies for the management of the disease. However, there is a large heterogeneity concerning the management of CF across centres. Standards have been proposed [30]. The stratification of CF patients is needed to characterise patients who 1) will require complete management in CF centres across their life cycle, 2) extensive diagnosis procedures and expensive treatment strategies, and 3) can be classified as having a milder phenotype. Innovative treatments may reverse certain severe sequelae of childhood diseases [31], but more information is needed to fully understand how innovative treatments may reverse apparently permanent lesions and to what extent.

\section{Networking}

Since 2008, the European Cystic Fibrosis Society (ECFS) has created, together with EuroCareCF, an international networking system, ECFS-Clinical Trial Network (ECFS-CTN), to develop clinical trials in order to improve the quality of life and treatment options for CF patients. The network includes 15 European Countries and $43 \mathrm{CF}$ centres with the aim of sharing expertise between healthcare professionals and patient organisations, and to develop new protocols in cooperation with the pharmaceutical industry. There is a close collaboration with a similar network in the USA (Cystic Fibrosis Foundation (CFF) Therapeutics Development Network).

\section{Care pathways}

Care pathways are essential. Improvements in the quality and implementation of medical care for individuals with CF have led to a dramatic improvement in survival. Many strategies have focused on the effective management of pulmonary disease, which has delayed the onset of a severe disease [32].

\section{mHealth}

The wide use of mobile phones is an opportunity to increase the autonomy of patients in the self-monitoring of their diseases and treatment (mHealth). This will create a faster communication 
channel with the CF reference centre, a regular capture of data by the patient to increase compliance, adapt the therapeutic strategy and improve the knowledge of the natural history of the diseases. However, there is insufficient evidence about the benefits of mHealth in CF [33].

\section{Importance of patients' associations}

Patient organisations have a triple role. First, they act as natural brokers for discussion with and between stakeholders in the healthcare system at the national level (e.g. Vaincre la Mucoviscidose in France, http://www.vaincrelamuco.org/) or with the European Commission; secondly, they are very important in financing research and linking the research with the healthcare system for knowledge transferability; and thirdly, patient organisations are essential for the organisation and recruitment of patients for clinical trials. They participate in the building of the community, therefore reducing isolation, they are a natural relay when families seek answers in medical and/or social care, and they help in research design etc.

\section{Patient empowerment}

A broader use of mobile technologies, in combination with the work of patient organisations in building the capacity of patients through therapeutic education programmes, leads to patient empowerment, which is a prerequisite to the development of a true patient-centric healthcare system. Therefore, well-informed patients can act as efficient co-decision makers with healthcare professionals and social workers as well as trusted partners, during the identification of patient-relevant outcomes (i.e. for the strategic approach of clinical trials) and patient-reported outcomes (i.e. to gather real-life evidence on medicines in development following adaptive pathways; patient registration; reported adverse effects during the pharmacovigilance phase and benefits in post-marketing evidence generation research plans, etc.).

\section{Patient health outcomes}

The AHA concept with a rare genetic disease, such as CF, implies the reorientation of the healthcare system towards a patient-centred approach, with empowered and autonomous patients. The system will then focus on the patient health outcomes notion in which the global health status is a key factor. This concept relies on patients themselves in collaboration with healthcare professionals, social workers and all stakeholders involved. The patient health outcomes are measured using the results that matter most to people when seeking care or treatment: prevention, recovery, functional improvement and the ability to live healthy and productive lives. There are challenges in the context of finite resources and societal preferences within a healthcare system. However, a number of specific activities, collaborations and innovations have recently emerged and require new forms of disruptive thinking from all healthcare stakeholders in Europe.

To support AHA, the healthcare systems need to be organised beyond the pill paradigm. Once the quality and efficiency of care (treatment and social) for chronic disease ageing patients is increased, avoidable hospitalisations are decreased through a patient-centred approach to care delivery.

\section{Social aspects of the strategy and policies}

Low socio-economic outcomes are associated with more severe CF [34]. Psychological interventions or approaches to support CF people and their caregivers are of importance, even though their benefit is still unclear [35]. Adolescents and children with CF report a sense of vulnerability, loss of independence and opportunities, isolation, and disempowerment. This reinforces the importance of the current model of multidisciplinary patient-centred care promoting shared decision making, control and self-efficacy, as well as physical and social functioning [36]. The new evolution of CF management is that adult patients are now experiencing the hope of an almost normal life. The psychological support of these patients is, therefore, very important to help them deal with this new concept of a long life with CF.

\section{Application of the pilot to other rare genetic diseases}

A holistic approach may help to promote AHA in CF patients. Longer and better lives represent progress and opportunities. The changes involved create major challenges for: individuals; employers; all public and private stakeholders, which include both health and social care; other welfare services; the governments; and all political parties.

$\mathrm{CF}$ is the most common genetic disease in Europe and the results obtained can be expanded to other rare genetic diseases, including some generic measures added to specific ones depending on the disease. The main goal is to start thinking about ageing during childhood (or even before conception). Results from the transition from paediatrics to elderly people will be of particular importance and will be carried out through the collaboration between the European Paediatric Society, the European Union Geriatric Medicine Society, the individual CF societies and patient organisations in the frame of the EIP on AHA i.e. MACVIA-LR. 


\section{References}

1 Döring G, Flume P, Heijerman H, et al. Treatment of lung infection in patients with cystic fibrosis: current and future strategies. J Cyst Fibros 2012; 11: 461-479.

2 Simmonds NJ. Ageing in cystic fibrosis and long-term survival. Paediatr Respir Rev 2013; 14: Suppl. 1: S6-S9.

3 Burgel PR, Bellis G, Olesen HV, et al. Future trends in cystic fibrosis demography in 34 European countries. Eur Respir J 2015; 46: 133-141.

4 Elborn JS, Bell SC, Madge SL, et al. Report of the European Respiratory Society/European Cystic Fibrosis Society task force on the care of adults with cystic fibrosis. Eur Respir J 2016; 47: 420-428.

5 Stephenson AL, Tom M, Berthiaume Y, et al. A contemporary survival analysis of individuals with cystic fibrosis: a cohort study. Eur Respir J 2015; 45: 670-679.

6 Zolin A, McKone EF, van Rens J, et al. ECFSPR Annual Report 2010. Karup, European Cystic Fibrosis Society, 2014. Available from: https://www.ecfs.eu/projects/ecfs-patient-registry/annual-reports

7 Kreindler JL, Miller VA. Cystic fibrosis: addressing the transition from pediatric to adult-oriented health care Patient Prefer Adherence 2013; 7: 1221-1226.

8 Schwarz C, Hartl D. Cystic fibrosis in Europe: patients live longer but are we ready? Eur Respir J 2015; 46: 11-12.

9 Samoliński B, Fronczak A, Wlodarczyk A, et al. Council of the European Union conclusions on chronic respiratory diseases in children. Lancet 2012; 379: e45-e46.

10 Bousquet J, Bourquin C, Augé P, et al. MACVIA-LR reference Site of the European Innovation Partnership on Active and Healthy Ageing (EIP on AHA) in Languedoc Roussillon. Eur Geriatr Med 2014; 5: 406-415.

11 Bousquet J, Jorgensen C, Dauzat M, et al. System medicine approaches for the definition of complex phenotypes in chronic diseases and ageing. From concept to implementation and policies. Curr Pharm Des 2014; 20: 5928-5944.

12 Bousquet J, Hajjam J, Piette F, et al. Les sites de reference Francais du Partenariat Européen d'Innovation pour un vieillissement actif et en bonne sante [The French reference sites of the European Innovation Partnership on active and healthy ageing]. Presse Med 2013; 42: 1558-1561.

13 Bousquet J, Anto JM, Berkouk K, et al. Developmental determinants in non-communicable chronic diseases and ageing. Thorax 2015; 70: 595-597.

14 Bousquet J, Kuh D, Bewick M, et al. Operative definition of active and healthy ageing (AHA): Meeting report. Montpellier October 20-21. Eur Geriatr Med 2015; 6: 196-200.

15 Bousquet J, Addis A, Adcock I, et al. Integrated care pathways for airway diseases (AIRWAYS-ICPs). Eur Respir J 2014; 44: 304-323.

16 Sermet-Gaudelus I, Mayell SJ, Southern KW, et al. Guidelines on the early management of infants diagnosed with cystic fibrosis following newborn screening. J Cyst Fibros 2010; 9: 323-329.

17 Southern KW, Mérelle MM, Dankert-Roelse JE, et al. Newborn screening for cystic fibrosis. Cochrane Database Syst Rev 2009: CD001402.

18 Ooi CY, Dupuis A, Ellis L, et al. Does extensive genotyping and nasal potential difference testing clarify the diagnosis of cystic fibrosis among patients with single-organ manifestations of cystic fibrosis? Thorax 2014; 69: 254-260.

19 Peckham D, Whitaker P. Drug induced complications; can we do more? J Cyst Fibros 2013; 12: 547-558.

20 Mall MA, Hartl D. CFTR: cystic fibrosis and beyond. Eur Respir J 2014; 44: 1042-1054.

21 Ramsey BW, Davies J, McElvaney NG, et al. A CFTR potentiator in patients with cystic fibrosis and the G551D mutation. N Engl J Med 2011; 365: 1663-1672.

22 Whiting P, Al M, Burgers L, et al. Ivacaftor for the treatment of patients with cystic fibrosis and the G551D mutation: a systematic review and cost-effectiveness analysis. Health Technol Assess 2014; 18: 1-106.

23 Boyle MP, Bell SC, Konstan MW, et al. A CFTR corrector (lumacaftor) and a CFTR potentiator (ivacaftor) for treatment of patients with cystic fibrosis who have a phe508del CFTR mutation: a phase 2 randomised controlled trial. Lancet Respir Med 2014; 2: 527-538.

24 Tiddens HA, Bos AC, Mouton JW, et al. Inhaled antibiotics: dry or wet? Eur Respir J 2014; 44: 1308-1318.

25 Langton Hewer SC, Smyth AR. Antibiotic strategies for eradicating Pseudomonas aeruginosa in people with cystic fibrosis. Cochrane Database Syst Rev 2014; 11: CD004197.

26 Brodt AM, Stovold E, Zhang L. Inhaled antibiotics for stable non-cystic fibrosis bronchiectasis: a systematic review. Eur Respir J 2014; 44: 382-393.

27 Kelly A, Moran A. Update on cystic fibrosis-related diabetes. J Cyst Fibros 2013; 12: 318-331.

28 Schwartz LA, Tuchman LK, Hobbie WL, et al. A social-ecological model of readiness for transition to adult-oriented care for adolescents and young adults with chronic health conditions. Child Care Health Dev 2011; 37: $883-895$.

29 de Manuel Keenoy E, David M, Mora J, et al. Activation of stratification strategies and results of the interventions on frail patients of healthcare services (ASSEHS) DG Sanco Project No. 201312 04. Eur Geriatr Med 2014; 5: $342-346$.

30 Conway S, Balfour-Lynn IM, De Rijcke K, et al. European Cystic Fibrosis Society Standards of Care: framework for the Cystic Fibrosis Centre. J Cyst Fibros 2014; 13: S3-S22.

31 Hayes D Jr, Long FR, McCoy KS, et al. Improvement in bronchiectasis on CT Imaging in a pediatric patient with cystic fibrosis on ivacaftor therapy. Respiration 2014; 88 : 345

32 Parkins MD, Parkins VM, Rendall JC, et al. Changing epidemiology and clinical issues arising in an ageing cystic fibrosis population. Ther Adv Respir Dis 2011; 5: 105-119.

33 Cox NS, Alison JA, Rasekaba T, et al. Telehealth in cystic fibrosis: a systematic review. J Telemed Telecare 2012; 18: 72-78.

34 Taylor-Robinson DC, Thielen K, Pressler T, et al. Low socioeconomic status is associated with worse lung function in the Danish cystic fibrosis population. Eur Respir J 2014; 44: 1363-1366.

35 Goldbeck L, Fidika A, Herle M, et al. Psychological interventions for individuals with cystic fibrosis and their families. Cochrane Database Syst Rev 2014; 6: CD003148.

36 Jamieson N, Fitzgerald D, Singh-Grewal D, et al. Children's experiences of cystic fibrosis: a systematic review of qualitative studies. Pediatrics 2014; 133: e1683-e1697. 